\section{LIMIT FOR THE PROPORTION OF REMAINING SAMPLES IN THE MODE- STIRRED CHAMBER SAMPLE SELECTION TECHNIQUE}

Paul Hallbjörner, ${ }^{1}$ Juan D. Sánchez-Heredia, ${ }^{2}$

Elias de los Reyes, ${ }^{3}$ and David A. Sánchez-Hernández ${ }^{4}$

${ }^{1}$ SP Technical Research Institute of Sweden, Box 857, SE-501 15

Borås, Sweden; Corresponding author: paul.hallbjorner@sp.se

${ }^{2}$ EMITE Ing, Edificio CEEIM, Campus Espinardo, E-30100 Murcia, Spain

${ }^{3}$ Departamento de Comunicaciones, Universidad Politécnica de

Valencia, Camino de Vera, s/n, E-46022 Valencia, Spain

${ }^{4}$ Departamento de Tecnologías de la Información y

Comunicaciones, Universidad Politécnica de Cartagena, Plaza del

Hospital, 1, E-30202 Cartagena, Spain

Received 25 January 2011

ABSTRACT: An expression is derived for the maximum proportion of remaining samples in the sample selection technique for mode-stirred chamber measurements. The limit is independent of the selection algorithm used. The limit is verified in simulations as well as measurement examples. (C) 2011 Wiley Periodicals, Inc. Microwave Opt Technol Lett 53:2608-2610, 2011; View this article online at wileyonlinelibrary.com. DOI 10.1002/mop.26336

Key words: mode-stirred chamber; reverberation chamber; sample selection; measurement technique

\section{INTRODUCTION}

Mode-stirred chamber (MSC) for antenna measurements is a well established technique, presented in several publications [14]. Recently, the sample selection technique [5-7] has been introduced for the purpose of improving the versatility of MSC measurements. In the sample selection technique, some measurement samples are selected while the rest are discarded, so that the selected sample set conforms to a predetermined probability density function (PDF). When applying the sample selection technique, the number of samples in a sequence decreases, and sometimes quite drastically. The number of remaining samples is one of the most critical parameters, and it should be as high as possible to achieve a good accuracy in the results. This article investigates the limit for the number of remaining samples in the sample selection technique.

\section{PROPORTION OF REMAINING SAMPLES}

The sample selection process will select a certain proportion of the initial (input) samples, that is, the number of remaining samples is linearly dependent on the number of initial samples. In the following, the proportion of remaining samples is studied, to make the results more general and applicable to initial sample sets of any length.

A function $f_{\text {initial }}(\mathbf{x})$ is used to describe the PDF of the initial data, and the target PDF after sample selection is defined by $f_{\text {tar- }}$ get $(\mathbf{x})$. Vector notation is used for the argument because multivariate distributions can be used, for instance for complexvalued amplitudes, or in measurements of multiple antennas. These functions have the usual properties of PDFs, that is, they are always positive and the integral over all arguments is unity. The total number of samples before and after sample selection is denoted as $N_{\text {initial }}$ and $N_{\text {target }}$, respectively. The number of samples within a small interval $\Delta \mathbf{x}$ at any $\mathbf{x}$ value is $N_{\text {initial }} f_{\text {initial }}(\mathbf{x}) \Delta \mathbf{x}$ and $N_{\text {target }} f_{\text {target }}(\mathbf{x}) \Delta \mathbf{x}$, respectively. Naturally, the number of samples at any $\mathbf{x}$ value can only remain the same or decrease as a result of the sample selection process. Consequently we have

$$
N_{\text {target }} f_{\text {target }}(\mathbf{x}) \leq N_{\text {initial }} f_{\text {initial }}(\mathbf{x}),
$$

where $\Delta \mathbf{x}$ is eliminated on both sides. As $f_{\text {target }}(\mathbf{x})$ is different from $f_{\text {initial }}(\mathbf{x})$ (the case when they are the same is trivial and not useful), there will be $\mathbf{x}$ values at which $f_{\text {target }}(\mathbf{x})>f_{\text {initial }}(\mathbf{x})$. To fulfill Eq. (1), $N_{\text {target }}$ then has to be smaller than $N_{\text {initial. }}$. It is the maximum ratio of $f_{\text {target }}(\mathbf{x}) / f_{\text {initial }}(\mathbf{x})$ for any $\mathbf{x}$ that dictates the necessary ratio of $N_{\text {target }} / N_{\text {initial }}$ for the condition in Eq. (1) to be satisfied. Defining the proportion of remaining samples $q$ as

$$
q=\frac{N_{\text {target }}}{N_{\text {initial }}}
$$

and substituting this in Eq. (1) gives the condition

$$
q \leq \frac{f_{\text {initial }}(\mathbf{x})}{f_{\text {target }}(\mathbf{x})}
$$

In a specific case, there can be some freedom in choosing $f_{\text {target }}(\mathbf{x})$, because PDFs often have one or several parameters and the requirements on $f_{\text {target }}(\mathbf{x})$ might not decide all of them. Remaining parameters should then preferably be set so that $q$ is optimized. The maximum value of $q$ is thus

$$
q_{\max }=\min \left(\frac{f_{\text {initial }}(\mathbf{x})}{f_{\text {target }}(\mathbf{x})}\right)
$$

for all $\mathbf{x}$, and for all $f_{\text {target }}(\mathbf{x})$ under the given constraints.

Different algorithms exist for the process of selecting samples [7]. The different algorithms have been reported to result in different proportions of remaining samples. The limit in Eq. (4) is arrived at without any assumptions regarding the algorithm used for selecting samples, so it is valid for any algorithm.

\section{APPLICATION EXAMPLES}

Two examples are provided in which the presented limit is used. In the first, it is showed that the so-called single-step algorithm [7] reaches $q_{\max }$. In the second, an expression for $q_{\max }$ is derived for a case of commonly used PDFs.

\subsection{Single-Step Algorithm}

The single-step algorithm works as follows: to decide whether a sample should be kept or not, it uses a threshold function $D(\mathbf{x})$, according to

$$
D(\mathbf{x})=C \frac{f_{\text {target }}(\mathbf{x})}{f_{\text {initial }}(\mathbf{x})},
$$

where $C$ is a constant. For each input sample at a value $\mathbf{x}_{k}$, a random number, uniformly distributed in the interval $[0$, $\max (D(\mathbf{x})))$, is generated. If the random number is less than $D\left(\mathbf{x}_{k}\right)$, the sample is kept. If it is greater than or equal to $D\left(\mathbf{x}_{k}\right)$, the sample is discarded.

The proportion of remaining samples after all input samples are covered is given by the multiple integral over the $n$-dimensional space of non-negative real numbers,

$$
q=\int_{\mathbf{x} \in \Re_{+}^{n}} D(\mathbf{x}) f_{\text {initial }}(\mathbf{x}) d \mathbf{x} .
$$


By substituting Eq. (5) into Eq. (6), and using the fact that the integral of a PDF is unity, it is found that

$$
q=C
$$

By letting $C=q_{\max }$, the single-step algorithm is optimal in terms of the proportion of remaining samples.

Because of the randomness by which the decision of selecting/discarding samples is taken, different realizations will result in different sets of selected samples, and also different numbers of remaining samples. The proportion of remaining samples in Eqs. (4) and (6) is exact for infinite length sequences, and accurate in the mean for finite length sequences.

\subsection{From Rayleigh to Rice}

An expression for $q_{\max }$ is derived for the case of having an initial data set with Rayleigh distributed amplitude, and a target PDF which the Rice distribution. In this example, complex-valued data samples, that is, amplitude and phase, are assumed. Each complex-valued data sample $z$ can be viewed as a twodimensional scalar sample $(x, y)$, related as

$$
z=x+j y .
$$

The bivariate normal distribution PDF [8] can be used for the initial data,

$$
f_{\text {initial }}(x, y)=\frac{1}{2 \pi s_{i}^{2}} \exp \left(-\frac{x^{2}+y^{2}}{2 s_{i}^{2}}\right) .
$$

By introducing an offset, it can represent Rice distributed scalar amplitudes, and it can therefore be used also for the target data. As phases are relative, and a constant phase offset applied to all data samples will not change the scalar amplitudes, the offset can for simplicity be chosen to be real valued. With the offset $a_{t}$,

$$
f_{\text {target }}(x, y)=\frac{1}{2 \pi s_{t}^{2}} \exp \left(-\frac{\left(x-a_{t}\right)^{2}+y^{2}}{2 s_{t}^{2}}\right) .
$$

Substituting Eqs. (9) and (10) into Eq. (4) results in the expression

$$
q_{\max }=\min \left(\frac{s_{t}^{2}}{s_{i}^{2}} \exp \left(\frac{\left(x-a_{t}\right)^{2}+y^{2}}{2 s_{t}^{2}}-\frac{x^{2}+y^{2}}{2 s_{i}^{2}}\right)\right) .
$$

To find the minimum, the derivatives of the argument with respect to $x$ and $y$ are calculated and set to zero. With the optimal values of $x$ and $y$,

$$
q_{\max }=\frac{s_{t}^{2}}{s_{i}^{2}} \exp \left(-\frac{1}{2}\left(\frac{a_{t}^{2}}{s_{i}^{2}-s_{t}^{2}}\right)\right)
$$

After this, the values of $a_{t}$ and $s_{t}$ that maximize $q_{\max }$ have to be chosen. Assume that a certain $K$-factor is desired in the target data, defined as

$$
K=\frac{a_{t}^{2}}{2 s_{t}^{2}}
$$

Using Eq. (13), Eq. (12) can be rewritten, derived with respect to $s_{t}$, and set to zero to find the optimal value of $s_{t}$.

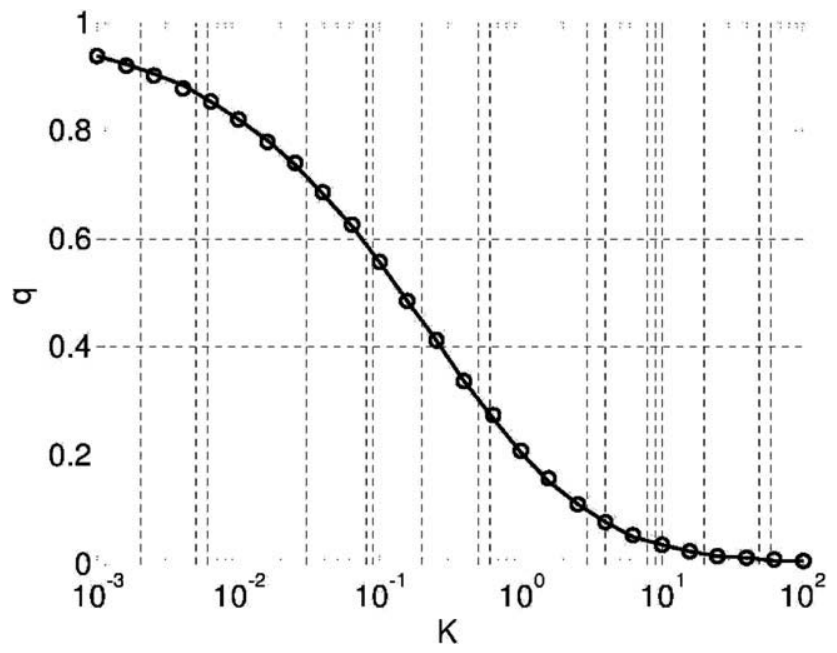

Figure 1 Proportion of remaining samples with the single-step algorithm (circles) and upper limit (solid line), when converting Rayleigh to Rice distribution using sample selection with simulated complex-valued data

The result is

$$
s_{t}=s_{i} \sqrt{1+\frac{K}{2}-\sqrt{\left(1+\frac{K}{2}\right)^{2}-1}}
$$

Substituting this expression into Eq. (12) gives

$$
q_{\max }=\left(1+\frac{K}{2}-\sqrt{\left(1+\frac{K}{2}\right)^{2}-1}\right) \exp \left(K+\frac{K}{\frac{K}{2}-\sqrt{\left(1+\frac{K}{2}\right)^{2}-1}}\right) .
$$

\section{VERIFICATION}

Numerical simulations in Matlab are used to verify Eq. (15). Random samples with Rayleigh distributed amplitude and uniform phase are generated. A sequence of 20,000 samples is

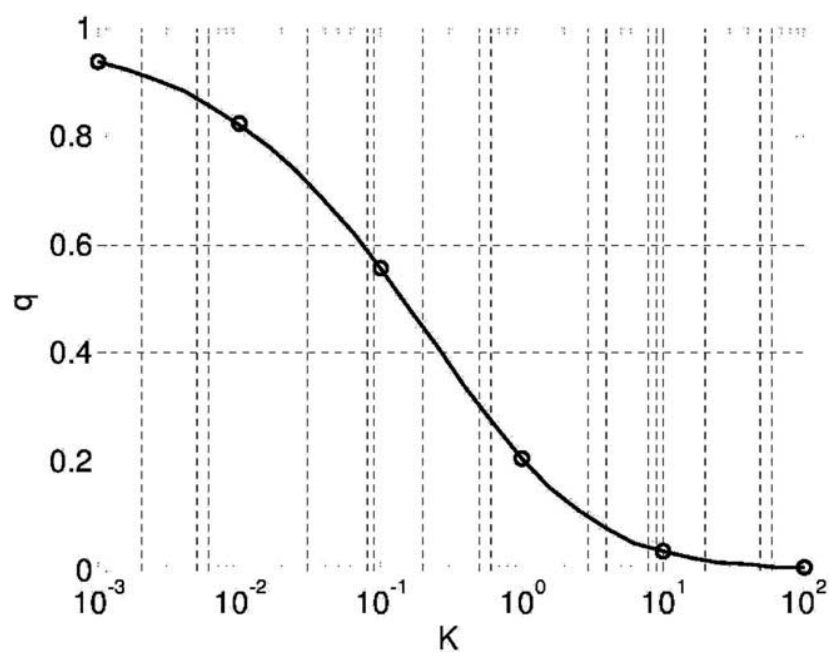

Figure 2 Proportion of remaining samples with the single-step algorithm (circles) and upper limit (solid line), when converting Rayleigh to Rice distribution using sample selection with measured complex-valued data 
generated, which is enough to make the statistical fluctuations due to the randomness of the single-step algorithm negligible. Sample selection is then applied, with the single-step algorithm, for a number of desired $K$-factors between 0.001 and 100 . Figure 1 shows the proportion of remaining samples from the simulations, together with the limit according to Eq. (15).

Measured samples are also used for verification. The singlestep algorithm is here applied to a sequence of 6400 samples, taken in a large MSC with mechanical mode stirring, at 2.6 $\mathrm{GHz}$. The test object is a single-element antenna with omnidirectional coverage. There is very little unstirred power in the chamber, making the raw data samples Rayleigh distributed. These results, together with Eq. (15), are shown in Figure 2.

\section{CONCLUSIONS}

An expression is derived for the upper limit of the proportion of remaining samples in the sample selection technique. The limit is independent of the algorithm used for the selection, and depends only on the PDFs of the initial and target data sets. Examples of how the limit can be used are given. The limit is verified experimentally in both simulations and measurements for the case of performing sample selection to achieve a Rice distributed data set from Rayleigh distributed initial data. Knowledge of the limit in practical situations will help estimating measurement uncertainty as well as enable assessment of the efficiency of sample selection algorithms.

\section{ACKNOWLEDGMENTS}

This work was supported in part by the Spanish National R\&D Programme through TEC2008-05811, and by Fundación Séneca, the R\&D coordinating agency for the Region of Murcia (Spain) under the $11783 / \mathrm{PI} / 09$ and $11610 / \mathrm{IV} 2 / 09$ projects.

\section{REFERENCES}

1. P. Corona, G. Latmiral, E. Paolini, and L. Piccioli, Use of a reverberating enclosure for measurements of radiated power in the microwave range, IEEE Trans Electromagn Compat EMC-18 (1976), 54-59.

2. T. Maeda and T. Morooka, Radiation efficiency measurement method for electrically small antennas using radio wave scatterers, IEEE AP-S Int Symp 1 (1988), 324-327.

3. J. Krogerus, K. Kiesi, and V. Santomaa, Evaluation of three methods for measuring total radiated power of handset antennas, In: IEEE Instrumentation and Measurement Technology Conference, Budapest, Hungary, May 21-23, 2001, pp. 1005-1010.

4. J.F. Valenzuela-Valdés, A.M. Martínez-González, and D.A. Sánchez-Hernández, Emulation of MIMO nonisotropic fading environments with reverberation chambers, IEEE Antennas Wireless Propag Lett 7 (2008), 325-328.

5. J.D. Sánchez-Heredia, M. Grudén, J.F. Valenzuela-Valdés, and D.A. Sánchez-Hernández, Sample-selection method for arbitrary fading emulation using mode-stirred chambers, IEEE Antennas Wireless Propag Lett 9 (2010), 409-412.

6. J.D. Sanchez-Heredia, M.A. Garcia-Fernandez, M. Grudén, P. Hallbjörner, A. Rydberg, and D.A. Sánchez-Hernández, Arbitrary fading emulation using mode-stirred chambers, EuCAP, in press.

7. M. Grudén, J.D. Sánchez-Heredia, P. Hallbjörner, J.F. ValenzuelaValdés, D.A. Sánchez-Hernández, and A. Rydberg, Sample selection algorithms for emulation of arbitrary fading distributions using modestirred chambers, IEEE Trans Electromagn Compat, submitted.

8. L. Råde and B. Westergren, Beta mathematics handbook for science and engineering, 3rd ed., Studentlitteratur, Lund, Sweden, p. 413, ISBN 91-44-25053-3.

(C) 2011 Wiley Periodicals, Inc.

\section{INDUCED ABERRATIONS BY COMBINATIVE CONVEX/CONCAVE INTERFACES OF REFRACTIVE-INDEX- MISMATCH AND CAPABILITY OF ADAPTIVE OPTICS CORRECTION}

\author{
Yiin Kuen Fuh, ${ }^{1,2}$ Kuo Chan Hsu, ${ }^{1}$ Jia Ren Fan, ${ }^{1}$ and \\ Ming Xien Lin ${ }^{1}$ \\ ${ }_{1}^{1}$ Institute of Opto-Mechatronics Engineering, National Central \\ University, No. 300, Jhongda Rd., Jhongli City, Taoyuan County \\ 32001, Taiwan; Corresponding author: mikefuh@cc.ncu.edu.tw \\ ${ }^{2}$ Department of Mechanical Engineering, National Central \\ University, No. 300, Jhongda Rd., Jhongli City, Taoyuan County \\ 32001, Taiwan
}

\section{Received 30 January 2011}

ABSTRACT: Adjustable fluidic adaptive lenses have been extensively studied because of the advantage of changing the geometric shape or refractive index without any mechanical moving parts. However, the induced aberrations were rarely discussed before. We address this issue by first clarifying the aberrations experimentally due to injected fluidic volumes. Experimental results show that under the injected fluidic volume of $0.1 \mathrm{ml}$, the main aberrations come from $Z_{1}$ (piston), $Z_{2}$ (tip), and $Z_{5}$ (defocus) and Zernike coefficients are 0.97, 0.31, and 1.31 $\mathrm{mm}$, respectively. In what follows, a series of tests specifically designed to explore the convex/concave interfaces and refractive-index-mismatch (RIM). Furthermore, we demonstrate the capability of adaptive optics (AO) correction on aberrations induced by combinative effects of multiple layers with convex/concave interfaces and RIM. A microelectromechanical system (MEMS) deformable mirror (DM) with 140 actuators was used in conjunction with Shack-Hartmann wavefront sensor to realize the experimentation. In particular, we consider the aberration introduced by interfaces of RIM between water/oil and glass. After AO correction, we can improve wavefront with root mean square (RMS) of 2.17-0.17 $\mu \mathrm{m}$ for an interface between water and glass. As for the interface between oil and glass, we are capable of improving RMS of 0.24-0.10 $\mu \mathrm{m}$. (C) 2011 Wiley Periodicals, Inc. Microwave Opt Technol Lett 53:2610-2615, 2011; View this article online at wileyonlinelibrary.com. DOI 10.1002/mop.26323

Key words: adaptive optics; refractive-index-mismatch (RIM); deformable mirror (DM); aberrations; Shack-Hartmann wavefront sensor

\section{INTRODUCTION}

Adaptive optics (AO) is a widely used concept and technology in astronomy to compensate the atmospheric turbulence that confines the performance of ground-based telescope [1-3]. In addition, the biological potentials such as correction of the ocular aberration for the improved in vivo imaging of the retina in vision science and ophthalmic applications are of recent thrust areas in research [4-9]. In microscopy, AO has also been applied to nonlinear microscopies such as multiphoton microscopy to image several layers of mismatched and inhomogeneous refractive index [10]. Moreover, the well-pursuit utilization of high numerical aperture (NA) objectives immersed in a fluid that is refractive index mismatched will inevitably induce the spherical aberration in the longitudinal direction [11]. AOassisted imaging system shows great capability of compensating spherical aberration at a certain depth in a specimen with refractive-index-mismatch (RIM) [12, 13].

Adjustable fluidic adaptive lenses have been studied recently with one significant advantage such that focal length can be easily altered by changing the geometric shape or refractive 